\title{
Elevation, moisture and shade drive the functional and phylogenetic meadow communities' assembly in the northeastern Tibetan Plateau
}

\author{
W. Qi ${ }^{1}$, X. Zhou ${ }^{1}$, M. Ma11, J. M. H. Knops ${ }^{2}$, W. Li ${ }^{1}$ and G. Dut1,3 \\ ${ }^{1}$ State Key Laboratory of Grassland Agroecosystems, School of Life Sciences, Lanzhou University, \\ South Tianshui Road 222, Lanzhou 730000, Gansu, People's Republic of China \\ ${ }^{2}$ School of Biological Sciences, University of Nebraska, Lincoln, NE 68588-0118, USA \\ ${ }^{3}$ Corresponding author.Email: guozdu@lzu.edu.cn
}

Keywords: Community assembly, Functional diversity, Phylogenetic diversity, Plant height, Specific leaf area, Seed mass.

\begin{abstract}
Despite a long history of alpine meadows studies, uncertainty remains about the importance of environmental factors in structuring their assembly. We examined the functional and phylogenetic structure of 170 alpine Tibetan meadow communities in relation to elevation, soil moisture and shade. Functional community structure was estimated with both communityweighted mean (CWM) trait values for specific leaf area (SLA), plant height and seed mass and functional diversity (Rao's quadratic index) for their traits individually and in combination (multivariate functional diversity). We found that shade induced by woody plants significantly increased the phylogenetic diversity and functional diversity of SLA of co-occurring species, suggesting that woody plants behave as "ecosystem engineers" creating a different environment that allows the existence of shade tolerant species and thereby facilitates the coexistence of plant species with different light resource acquisition strategies. We also found evidence for a clear decrease in phylogenetic diversity, CWM and functional diversity related to plant height in the two extreme, both the dry and wet, soil moisture conditions. This indicates that both drought and excess moisture may act as environmental filters selecting species with close phylogenetic relationships and similar height. Moreover, we detected significant decreases in both CWM and functional diversity for seed mass along elevational gradients, suggesting that low net primary productivity (NPP) limits seed size. Finally, because of different individual trait responses to environmental factors, the multivariate functional diversity did not change across environmental gradients. This lack of multivariate response supports the hypothesis that multiple processes, such as environmental filtering, competition and facilitation, may operate simultaneously and exert opposing effects on community assembly along different niche (e.g., water use, light acquisition) axes, resulting in no overall functional community structure change. This contrast between individual and multivariate trait patterns highlights the importance of examining individual traits linked with different ecological processes to better understand the mechanisms of community assembly.
\end{abstract}

Nomenclature: Wu and Raven (1994-2013).

Abberviations: CWM-Community Weighted Mean, L-H-S-Leaf-Height-Seed Scheme, MPD-Mean Phylogenetic Distance, NPP-Net Primary Productivity, SES- Standardized Effect Size, SLA-Specific Leaf Area.

\section{Introduction}

Understanding community assembly processes is a central theme in plant ecology (Chesson 2000, Ricklefs 2004). Community assembly may be driven by random processes associated with disturbance and local and regional species pool demographic dynamics (Hubbell 2001, Dornelas et al. 2006), and/or deterministic processes, such as environmental filtering and competition (Cornwell and Ackerly 2009, Adler et al. 2013). Both environmental filtering and competition are linked to species' niches and can drive assembly processes in opposite directions. For example, the physical environment generally imposes an ecological and evolutionary 'filter' such that species with similar ecological requirements are found in similar environments, a pattern referred to as spatial niche clustering (Weiher et al. 1998, Duarte 2011). Competition is likely to have the opposite effect, limiting similarity of species (MacArthur and Levins 1967, Yang et al. 2012). In addition, several other processes such as equalizing fitness processes and facilitation also can affect community assembly (Chesson 2000, Grime 2006, Baumeister and Callaway 2006). Ultimately, patterns of species coexistence may depend on how these assembly processes act over ecological and evolutionary time-scales (Webb et al. 2002, Baraloto et al. 2012).

If two species niches overlap, it is expected that these species also have similar ecological strategies for survival, growth, reproduction, resource capture and defense (Westoby and Wright 2006, Yang et al. 2012). Since plant functional traits influence ecological strategies (McGill et al. 2006), functional traits have been used to examine community assembly (Ackerly 2003, Baraloto et al. 2012). Westoby (1998) proposed three functional traits namely specific leaf area (SLA), plant height and seed mass as a simple 'Leaf-Height- 
Seed' (L-H-S) scheme that operationally quantifies a plant species ecological strategy. SLA is correlated with other plant traits such as photosynthetic leaf $\mathrm{N}$ content, leaf life-span and relative growth rate, representing, therefore, a trade-off between the leaf carbon acquisition rate and the longevity of plant tissues (Westoby 1998). Plant height represents a tradeoff associated with the competitive ability to reach higher in a canopy and thereby intercept more light and the biomass cost for the supporting structure needed to reach this canopy position. Seed mass signifies the trade-off between dispersal probability and a seedling establishment's ability (Lloret et al. 2004). Community-level L-H-S trait distribution patterns (e.g., clustering or divergence) can disentangle the importance of these trade-offs during community assembly (Pillar et al. 2009) and help predict how assembly processes respond to environmental changes.

It is also important to incorporate phylogenetic information into trait-based assembly processes analyses (Silvertown et al. 2006, Duarte 2011), because, firstly, some functional traits are phylogenetically conserved. Thus, these trait values are not randomly distributed, but tend to covary based on the degree of phylogenetic relatedness among species (Wiens and Graham 2005, Yang et al. 2012). Then, it is difficult to measure each functional trait for all species in a multi-site or multi-community study. Phylogenetic trees, if they capture phylogenetically correlated functional traits, can be used as a proxy for difficult-to-measure functional traits (Baraloto et al. 2012). Finally, species' phylogenetic information can reflect the historical factors related to biogeographical and macroevolutionary processes (Leibold et al. 2010). These historical factors largely determine the composition of regional species pools, and thus probably influence the responses of plant communities to environmental changes (Crisp et al. 2009, Yang et al. 2012).

In alpine areas, elevation is the most striking factor associated with both plant distributions and biodiversity patterns (Wang et al. 2007, Qi et al. 2014b). However, functional and phylogenetic structure of plant communities have shown contrasting patterns along elevational gradients. For example, Kluge and Kessler (2011) discovered that functional traits of epiphytic fern communities tended to be clustered at high elevations suggesting environmental filtering in stressful environment, and overdispersion of functional traits at middle elevations suggesting strong interspecific competition in benign environments. Similarly, Li et al. (2014) found that with increasing elevation from 3000 to $5500 \mathrm{~m}$ in the Hengduan Mountains of China, phylogenetic community structure changed from overdispersion to clustering. In contrast, Bryant et al. (2008) found that plant communities in Rocky Mountain of Colorado increase in phylogenetically overdispersion with increasing elevation. Swenson et al. (2011) similarly described an increase in functional diversity with elevation in the Luquillo Forest of Puerto Rico.

Similarly to elevation, soil moisture conditions can correlate strongly with functional and/or phylogenetic community structure (Karst et al. 2005, Bernard-Verdier et al. 2012), but contrasting patterns have emerged. For example, Kembel and Hubbell (2006) discovered that low soil moisture can act as environmental filter selecting for drought-tolerant species and thereby producing phylogenetic clustering in neotropical forest communities. Kluge and Kessler (2011) also found a significant functional clustering of epiphytic fern communities in arid environments. However, Katabuchi et al. (2012) reported different patterns that along soil moisture gradients with-community variances and ranges were reduced for seed mass, increased for some leaf traits (SLA, leaf size and leaf nitrogen content), but non-significantly varied for multivariate trait combinations. Cornwell et al (2006) also found no difference in multivariate trait diversity between wet and dry chaparral communities, even though species in drier (or wetter) communities exhibited greater diversity in SLA and wood density (or seed mass).

Shade induced by tall woody species is frequently reported to increase the phylogenetic and functional diversity of plant communities because woody species may act as an "ecosystem engineer" creating a novel environment that allows for the coexistence of species with distant phylogenetic relatedness and different traits (Tirado and Pugnaire 2005, Baumeister and Callaway 2006, Valiente-Banuet and Verdú 2007). However, some studies (Valladares and Niinemets 2008, Willis et al. 2010) found a strong phylogenetic signal in both species light availability niche and relevant functional traits. Thus, it is expected that shade induced by woody species may also act as environmental filters selecting for species with similar light availability (e.g., shade-tolerant) niche and its relevant functional traits, resulting in functional and phylogenetic clustering for understory plant communities.

Meadow vegetations cover about $25 \%$ of the surface of the Tibetan Plateau, and the geographic distribution of these meadows is largely determined by regional or local climate, elevation, and soil properties (CVEC, CAS 1980). Tibetan meadows are among the most impacted ecosystems facing the selection pressures caused by extreme climate (Klein et al. 2007, Yang et al. 2012), making they an ideal system to examine the dynamics and mechanisms driving plant community assembly. Here, we use both an L-H-S trait data set and a phylogenetic tree to test functional and phylogenetic assembly scenarios and test (i) whether functional diversity is low in stressful environments (e.g., low soil moisture or high elevation) and increases in more benign environments as competition becomes more important, (ii) whether shade induced by woody species enhances community functional diversity, because of the increase habitat variability, or alternatively if shade induced by woody species increases environmental stress and thereby increases the functional or phylogenetic meadow species similarity.

\section{Materials and methods}

\section{Study area}

The study area was located on the northeast edge of the Tibetan Plateau $\left(101^{\circ} 05^{\prime}-104^{\circ} 40^{\prime}\right.$ E, $\left.32^{\circ} 60^{\prime}-35^{\circ} 30^{\prime} \mathrm{N}\right)$. Meadow vegetations are present between $2700 \mathrm{~m}$ and $4100 \mathrm{~m}$ of this region. Using a principal component analysis (PCA) of 
seven climatic variables from the 10 nearest weather stations (Table S1) and a Pearson correlation between PCA scores and elevation, we found that elevation was significantly associated with PCA axis $1(\mathrm{r}=-0.937, \mathrm{P}<0.001)$, the axis associated with temperature-related variables. Elevation was not correlated with PCA axis $2(r=0.136, P=0.708)$, the axis associated with moisture-related variables (Fig. S1). Thus, for this location, elevation represents a temperature and not a moisture availability gradient. The geographic distribution of meadow communities within each elevational level also depends largely on soil moisture conditions (Table S2). In addition, meadow community composition is influenced by the presence of trees and shrubs, such as Hippophae, Salix and Lonicera species (CVEC, CAS 1980).

\section{Field sampling}

We sampled 57 sites in 2008 or 2009 (Appendix 1), which located at each $100-\mathrm{m}$ interval between $2700 \mathrm{~m}$ and $4100 \mathrm{~m}$. All sampling was conducted in meadows that are used as winter time pasture for yak and sheep. The elevational distribution of sampled sites was unimodal, with a peak in $3300 \mathrm{~m}$ $-3600 \mathrm{~m}$, because a larger land area is present at this elevation, which also causes more different species and meadow subtypes at this elevation. At each site we placed three 0.5 $\mathrm{m} \times 0.5 \mathrm{~m}$ quadrats at locations that differed in soil moisture conditions and, if present, shade (one site had only two quadrats because of homogeneous soil moisture and shade; thus altogether 170 quadrats were established). In each quadrat, the percent cover of each species was recorded at the peak standing biomass in late July and August.

In each quadrat, we sampled soil to a $30 \mathrm{~cm}$ depth in 2 or 3 depth intervals of $15 \mathrm{~cm}$ or $10 \mathrm{~cm}$. Soil moisture content (a percentage of soil dry weight) of each depth interval was measured gravimetrically by drying the soil at $105^{\circ} \mathrm{C}$ for 24 $\mathrm{h}$ and then averaged the quadrat's soil moisture content. We failed to measure soil moisture content in 10 quadrats. Shade was defined as the area under or next to a tree or a shrub, where more than $80 \%$ sunlight does not reach the ground during most of a day.

\section{Trait measurement}

Altogether we recorded 271 herbaceous species which accounted for more than $95 \%$ of the aboveground biomass and plant cover and we used 271 as the regional species pool. The trait measurement methods were described in Qi et al (2014a) and Appendix 2 (trait data see Appendix 3).

\section{Data analyses}

We used the community-weighted mean (CWM) trait and the Rao quadratic diversity $(\mathrm{Q})$ to estimate the functional community structure. The CWM trait represents the average of the functional traits within a community, while the Q can be viewed as a measure of trait dispersion (diversity) within the community (Lavorel et al. 2008, Carboni et al. 2013).
Combined these two indices can effectively describe two different aspects of the species trait turnover along environmental gradients (Ricotta and Moretti 2011, Carboni et al. 2013): changes in the CWM trait can be used to analyze environmental filtering in relation to functional traits, while shifts in Q reflect trait dispersion patterns in relation to community assembly. Also, Q can be used to assess in multiple trait space, the combined role of functional traits in community assembly. We calculated CWM trait values and Q for each L-H-S trait individually and $\mathrm{Q}$ for all traits in combination by using FD package in R (R Development Core Team 2010).

Before phylogenetic community analyses, a working phylogenetic tree was generated using 'Phylomatic' (Webb et al. 2008) in association with the ' $R 20120829$ ' version of the Angiosperm Phylogeny Group III data (available at http://www.phylodiversity.net). Branch lengths according to Wikström et al. (2001) were assigned using the BLADJ algorithm (Webb et al. 2008).

To test whether we needed to account for phylogenetic community, we examined the phylogenetic signal strength by estimating Pagel's $\lambda$ for each L-H-S trait using "fitContinuous" functions in the R package "geiger" v2.0.3 (Pagel 1999, Harmon 2014). We used a maximum likelihood framework to estimate the parameter $\lambda$ (phylogenetic signal value), which can vary from 0 (no influence of phylogeny) to 1 (maximum phylogenetic influence). Approximate confidence intervals for the maximum likelihood value of $\lambda$ were calculated via likelihood ratio tests (Freckleton et al. 2002).

When a phylogenetic signal existed in a L-H-S trait (i.e. $\lambda$ was significantly different from 0 ), we calculated the mean phylogenetic distance (MPD) using the 'comstruct' function in Phylocom-4.1 to quantify the phylogenetic relatedness of co-occuring species (Webb et al. 2008). Communities that are phylogenetically overdispersed have MPD values higher than expected by chance, whereas communities that are phylogenetically clustered have MPD values lower than expected by chance.

To determine whether the pattern in MPD and $Q$ of a community was significantly different from the null distribution at $a=0.05$ (two-tails), we calculated a standardized effect size (SES) by generating 9999 random assemblages for each community, with preserving the relative abundance patterns and shuffling the taxon names. For MPD, we calculated $\mathrm{SES}_{\mathrm{M}}$ as: $\mathrm{SES}_{\mathrm{M}}=\left(\mathrm{MPD}_{\mathrm{OBS}}-\mathrm{MPD}_{\text {RANDOM }}\right) / \mathrm{sd}\left(\mathrm{MPD}_{\mathrm{RANDOM}}\right)$, where $\mathrm{sd}\left(\mathrm{MPD}_{\text {RANDOM }}\right)$ is the standard deviation of the 9999 random MPD values. Thus, $\mathrm{SES}_{\mathrm{M}}$ is equivalent to $-1 \times \mathrm{NRI}$ (net relatedness index, Webb et al. 2002). For $Q$, we calculated $\mathrm{SES}_{\mathrm{Q}}$ as: $\mathrm{SES}_{\mathrm{Q}}=\left(Q_{\mathrm{OBS}}-Q_{\mathrm{RANDOM}}\right) / \mathrm{sd}\left(Q_{\mathrm{RANDOM}}\right)$. A positive/negative $\mathrm{SES}_{\mathrm{Q}}$ or $\mathrm{SES}_{\mathrm{M}}$ indicates that traits or phylogeny are dispersed/clustered within a community; and if the value of $\mathrm{SES}_{\mathrm{Q}}$ or $\mathrm{SES}_{\mathrm{M}}$ for a community is higher than 1.96 (or lower than -1.96), a significant functional or phylogenetic overdispersion (or clustering) is found (Vamosi et al. 2009). Moreover, we used a one sample $t$-test to examine whether $\mathrm{SES}_{\mathrm{M}}$ and $\mathrm{SES}_{\mathrm{Q}}$ of each L-H-S trait and multivariate traits presented significant deviations from a null expectation $($ mean $=0)$. 
We used one-way ANOVA (analysis of variance) to test for the differences in response variables (e.g., CWM of L-H-S trait values, $\mathrm{SES}_{\mathrm{M}}$, and $\mathrm{SES}_{\mathrm{Q}}$ of each L-H-S trait and multivariate traits) for the shade and no shade locations. We performed both linear and quadratic regressions to examine the relationships between elevation or soil moisture content and response variables. The one-way ANOVA and regression analysis were conducted using SPSS 16.0 software (Chicago, IL, USA).

\section{Results}

We found a significant phylogenetic signal in all three of the L-H-S traits (Table 1), with the stronger signal for seed mass $(\lambda=0.989)$, followed by SLA $(\lambda=0.526)$ and plant height $(\lambda=0.429)$.

Both the linear and quadratic regression showed that the SLA and plant height significantly decreased, and seed mass showed no relationship, with elevation (Fig. 1A). Along soil moisture content gradient, we found that there was no relationship for SLA, and both the linear and quadratic regression showed a decrease in plant height and seed mass with increasing soil moisture content (Fig. 1B). In contrast, there was no significant difference between shaded and non-shaded communities in CWM height (95\% confidence intervals: -0.692 --0.645 and $-0.753--0.655$, respectively, log-scale, hereinafter), SLA (2.196-2.211 and $2.193-2.224)$, and seed mass $(-0.076--0.020$ and $-0.101-0.015)$ (figures not shown).

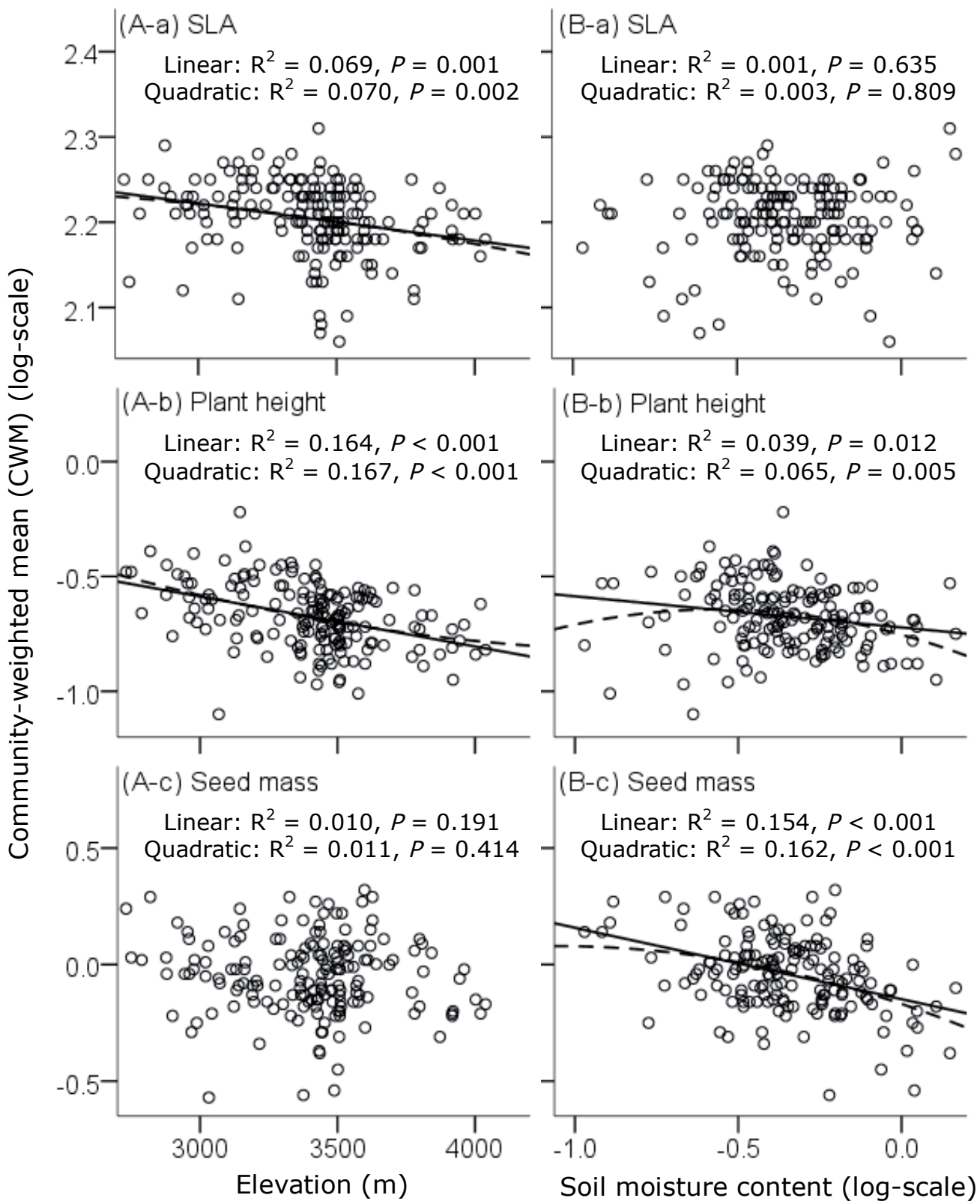

Figure 1. Community-weighted mean (CWM) specific leaf area (SLA, a, $\mathrm{cm}^{2} / \mathrm{g}$ ), plant height (b, m), seed mass (c, mg) in relation to the elevation (A) and soil moisture (B) Significant linear and quadratic relationship (at $\alpha=0.05$ ) are shown as solid line and dashed line, respectively. 
Table 1. Phylogenetic signal of functional traits of meadow species phylogeny on the northeastern Tibetan Plateau.

\begin{tabular}{cccccc}
\hline \hline Functional traits & $\begin{array}{c}\text { Number of } \\
\text { species }\end{array}$ & $\lambda$ & $95 \%$ CI & \multicolumn{2}{c}{ Significance of the difference from } \\
\cline { 3 - 5 } & 271 & 0.526 & $0.117-0.821$ & 0.004 & $\lambda=1$ \\
\hline Specific leaf area & 265 & 0.429 & $0.171-0.720$ & $<0.001$ & $<0.001$ \\
Plant height & 271 & 0.989 & $0.975-0.995$ & $<0.001$ & $<0.001$ \\
Seed mass & & & & \\
\hline
\end{tabular}

Note: the maximum likelihood value of $\lambda$ was given along with confidence intervals for this value, and the significance (P-values) of the difference from $\lambda=0$ and $\lambda=1$.

Figure 2. The standardized effect size (SES) of Rao quadratic diversity $(Q)$ for the specific leaf area (SLA, a), plant height (b), seed mass (c), multivariate traits (d), and phylogenetic diversity (e) in relationship to the elevation (A) and soil moisture content (B). Positive (or negative) values indicate greater (or lower) functional and phylogenetic diversity than null. Symbols in black represent communities where functional or phylogenetic diversity significantly differed from the null expectation. Significant linear and quadratic relationship (at $\alpha=0.05$ ) were shown as solid line and dashed line, respectively.

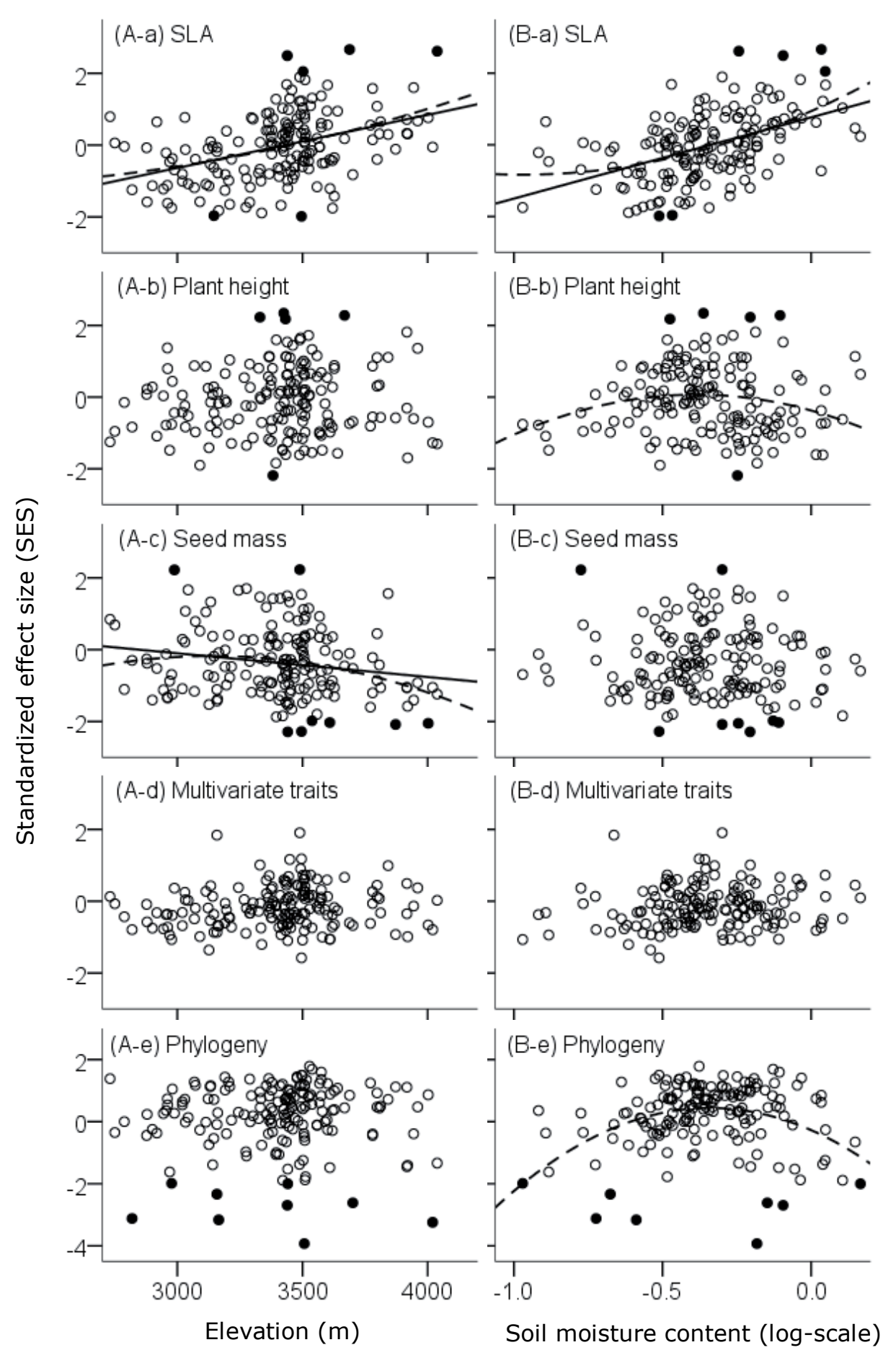




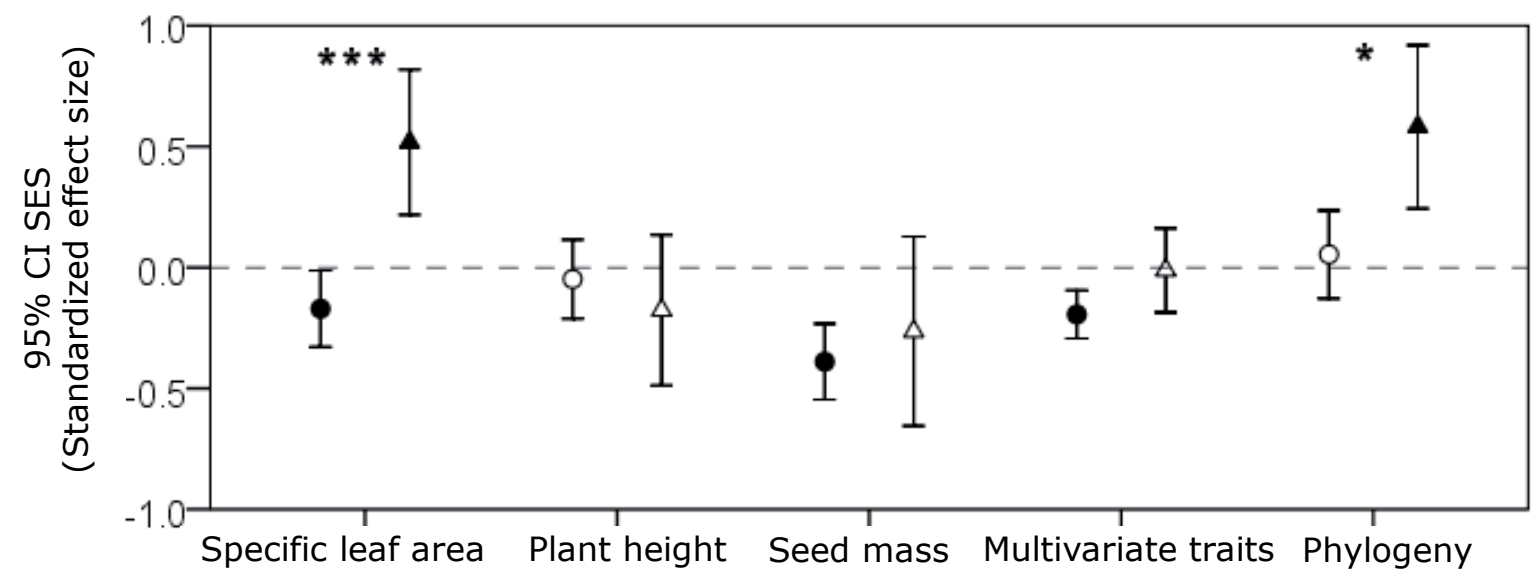

Figure 3. Standardized effect size (SES) of Rao quadratic diversity $(Q)$ for each L-H-S trait and the multivariate traits and phylogenetic diversity for the shade and non shade condition ( $\circ$, shade; $\Delta$, non-shade). Shown are the means \pm 95 CI. Symbols in black indicate values significantly different from zero. $* * * P<0.001, * * P<0.01, * P<0.05$.

Single trait functional diversity varied from significantly less (clustering), to not significance, to significantly greater than expected (overdispersion) from the null expectation. We found significant functional clustering for SLA in 2, plant height in 1 and seed mass in 6 communities, and significant functional overdispersion for SLA in 4, plant height in 4 and seed mass in 2 communities, respectively (Fig. 2). In contrast, functional diversity in multivariate trait space was never lower nor greater than the null expectation; while phylogenetic diversity was lower than expected in nine communities (Fig. 2).

Considering all meadow communities, the mean $\mathrm{SES}_{\mathrm{M}}$ was higher, whereas the mean $\mathrm{SES}_{\mathrm{Q}}$ of each L-H-S trait and multivariate traits, were all lower than zero, indicating an overall phylogenetic overdispersion and a functional clustering within a community. However, based on $95 \%$ confidence intervals $(\mathrm{CI}), \mathrm{SES}_{\mathrm{Q}}$ of SLA (95\% CI: $\left.-0.186-0.104\right)$ and plant height (95\% CI: $-0.216-0.072)$ were non-significantly; and the $\mathrm{SES}_{\mathrm{M}}(95 \% \mathrm{CI}:-0.009-0.316)$ was only marginally significantly different $(P=0.064$; Fig. S2).

We did not find a significant relationship between elevation and $\mathrm{SES}_{\mathrm{Q}}$ of plant height (Fig. 2A-b). The relationship between elevation and $\mathrm{SES}_{\mathrm{Q}}$ of SLA, however, did show both a significant monotonic increase (Fig. $2 \mathrm{~A}-\mathrm{a}, \mathrm{R}^{2}=0.168, P$ $<0.001$ ) and U-shaped curve (Fig. 2A-a, $\mathrm{R}^{2}=0.167, P<$ $0.001)$. In contrast, the relationship between elevation and $\mathrm{SES}_{\mathrm{Q}}$ of seed mass showed both a significant monotonic decrease (Fig. 2A-c, $\mathrm{R}^{2}=0.033, P=0.018$ ) and unimodal curve (Fig. 2A-c, $\mathrm{R}^{2}=0.053, P=0.010$ ). Because of these contrasting patterns, the multivariate trait space functional diversity did not change across elevational gradients (Fig. 2Ad). Finally, there was also no significant linear or quadratic relationship between elevation and $\mathrm{SES}_{\mathrm{M}}$ (Fig. 2A-e).

We also found significantly different L-H-S traits for the $\mathrm{SES}_{\mathrm{Q}}$ - soil moisture content relationship. The $\mathrm{SES}_{\mathrm{Q}}$ of SLA increased significantly monotonic (Fig. $2 \mathrm{~B}-\mathrm{a}, \mathrm{R}^{2}=0.239, P$ $<0.001$ ) and U-shaped with soil moisture content (Fig. 2B-a, $\left.\mathrm{R}^{2}=0.255, P<0.001\right)$. In contrast, the relationship between soil moisture content and $\mathrm{SES}_{\mathrm{Q}}$ of plant height was only sig- nificantly unimodal (Fig. 2B-b, $\mathrm{R}^{2}=0.043, P=0.031$ ). For seed mass, we found no significant (Fig. 2B-c) relationship between soil moisture content and $\mathrm{SES}_{\mathrm{Q}}$. Similarly to elevation, because of these contrasting patterns, multivariate trait space functional diversity did not change across the soil moisture gradients (Fig. 2B-d). Finally, the relationship between soil moisture content and $\mathrm{SES}_{\mathrm{M}}$ was only significantly unimodal (Fig. 2B-e, $\mathrm{R}^{2}=0.154, P<0.001$ ).

The shaded communities had significantly higher $\mathrm{SES}_{\mathrm{Q}}$ of SLA, and non-shaded communities had significantly lower $\mathrm{SES}_{\mathrm{Q}}$ of SLA than zero (Fig. 3). Shading did not influence the $\mathrm{SES}_{\mathrm{Q}}$ of plant height and seed mass, except that the $\mathrm{SES}_{\mathrm{Q}}$ of seed mass of the non-shaded communities was significantly lower zero (95\% CI: $-0.114--0.053$, Fig. 3). Considering all traits, the functional diversity was not significantly different between shaded and non-shaded communities. Lastly, phylogenetic diversity was significantly higher in shaded versus non shaded communities, where the $\mathrm{SES}_{\mathrm{M}}$ of the shaded communities was significantly different than zero (Fig. 3).

\section{Discussion}

Tibetan meadows showed highly divergent L-H-S traits in relation to elevation, soil moisture and shade. Specifically, the functional diversity of SLA significantly increased with elevation, with soil moisture content and with shade. Plant height showed a significant unimodal trend in its community mean and functional diversity with soil moisture; whereas seed mass significantly decreased in its community mean with soil moisture but in its functional diversity with elevation. However, the multivariate functional diversity of all traits together did not change over these environmental gradients. Moreover, phylogenetic diversity was significantly higher in shade and in moderate soil moisture. Combined, these results indicate that different assembly processes, such as abiotic filtering, equalizing fitness processes, facilitation and competition, may structure these communities. 


\section{Overall patterns in functional and phylogenetic diversity}

Our analysis indicated an overall patterns of phylogenetic overdispersion, but functional clustering in the individual and multivariate trait space. A degree of random functional trait distribution within the phylogeny (i.e. Pagel's $\lambda$ of L-H-S traits, especially SLA and plant height, are significantly different from 1) may have caused this inconsistency (Webb et al. 2002, Kraft et al. 2007). When closely related species coexist, they may undergo trait shifts ('character displacement'; Schluter, 2000), thereby reducing niche overlap. Other traits strongly driven by environmental conditions such as small leaves and low SLA driven by drought and disturbance, may cause similar trait values in coexisting plant species from distantly related clades (Ackerly 2004).

Environmental filtering and equalizing fitness processes may contribute to the overall functional clustering. As is commonly assumed in community assembly, environmental habitat filtering may cause the assembly of species with similar functional traits because these traits are optimal for this environment (Weiher and Keddy 1995, Cornwell and Ackerly 2009, Lebrija-Trejos et al. 2010). Our findings of the community-weighted height and SLA (or height and seed mass, respectively) significantly decrease with increasing elevation (or soil moisture) support such environmental habitat filtering. Alternatively, our results can also be explained by equalizing fitness processes where resource competition constrains a community to certain traits or trait combinations, which causes greater niche overlap and functional redundancy around an optimal trait (Grime 2006, Spasojevic and Suding 2012, Lewis et al. 2014).

\section{Change in individual functional community traits along gradients}

Elevation. Overall, we found an SLA functional diversity increase with elevation, which supports the hypothesis of increased SLA divergence among co-occurring species with increasing environmental stresses such as low temperature, strong wind and high solar radiation. In high-elevation (alpine) zones, species with leaves in the upper canopy such as Kobresia and Carex have thick and tough (low SLA) leaves as an adaptation to such environmental stresses. The canopy of these species can create an environment with benign conditions (e.g., low wind speed and low solar radiation) for species in bottom layer (i.e. the species growing near ground) and promotes them (e.g., Polygonum, Viola) to develop thin leaves. In low-elevation (temperate) zones, however, lower stress allow species in both the upper and lower canopy layers to develop thin leaves (Hakes and Cronin 2011), resulting in a higher community-weighted SLA mean and lower SLA diversity. In contrast, the response of $\mathrm{SES}_{\mathrm{Q}}$ and community-weighted seed mass to elevation was negative, which is consistent with our previous findings that seed mass tends to converge around a low value along elevation gradients, supporting the hypothesis that decreasing net primary productivity (NPP) does not allow for large seed to ensure enough seed production (Du and Qi 2010, Qi et al. 2014a). Lastly, the significantly negative association of elevation with CWM plant height, but not with $\mathrm{SES}_{\mathrm{Q}}$ of plant height, supports the hypothesis that with increasing elevation coexisting meadow species generally develop small stature as an adaption to the decrease in NPP, and maintain similar plant height dispersion to obtain suitable niche partitioning (e.g., limiting similarity) along light competition axis.

Soil moisture. Similar as elevation, the relationships between soil moisture and phylogenetic and functional structure is also complex. The phylogenetic diversity decreased in communities under moderate soil moisture (phylogenetic overdispersion) as compared to communities under lower or higher soil moisture content (phylogenetic clustering). Plant height matches this phylogenetic pattern and may reflect an overall selection for smaller plant size both in arid habitats, to reduce vessel length resistance to water transport (Westoby 1998, reviewed by Westoby et al. 2002), and in water-excess habitats (e.g., swamp) as a response to deficient soil aeration (Cornwell et al. 2006, Gross et al. 2008). The hypothesis is supported by our significant unimodal relationship between CWM plant height and soil moisture. In contrast, the overall SLA diversity increase with increasing soil moisture, may be explained by the fact that high-SLA $\left(>230 \mathrm{~cm}^{2} / \mathrm{g}\right)$ species can be associated both with high transpiration rates or with a low drought tolerance (Westoby et al. 2002, Gibbs and Greenway 2003, Bailey-Serres and Voesenek 2008), causing a narrow range of SLA value in arid and semiarid meadow communities.

Shade. We did not find any significant influence of shade on community-weighted L-H-S traits. The non-shaded communities, however, were composed of species more similar in their phylogeny and SLA than the shaded communities. Similar findings have been observed in other communities around the world. In Mexican semi-arid woodlands, for instance, woody species facilitated the co-occurrence of distantly related species and increased phylogenetic community diversity (Valiente-Banuet and Verdú 2007). Successional community studies also have found that the mean phylogenetic distance of co-occurring species increases over time after the establishment of woody shaded species (Verdú et al. 2009, Norden et al. 2012).

The increase in phylogenetic and functional diversity under shaded condition may be explained by two mechanisms: a "nurse plant" facilitation effect, in which tall canopy species create an environment that allows species that have a sensitive life stage as seedlings to successfully establish and become part of a plant community, or, an "ecosystem engineer" facilitation, in which tall canopy plants create a different, more benign environment that allows plant species with different traits to establish and complete their entire life history. Both these facilitations can lead to (i) an increase in biological diversity by ameliorating the harsh alpine environment (Valiente-Banuet and Verdú 2007), and (or) (ii) expand the realized niches of less stress tolerant species when climate is stressful (Baumeister and Callaway 2006). We collected all our data on mature plants, thus our data fits best with the "ecosystem engineer" facilitation. However, we did not detect any shade effect on the within-community seed mass and 
plant height dispersion. Shade may also acts as an environmental stress selecting species with a similar large seed mass, to allow seedling establishment, and large height, to allow access to the top of the canopy (Westoby et al. 2002, Myers and Kitajima 2007). Thus, in contrast to SLA, seed size and plant height do not fit within an "ecosystem engineer" facilitation hypothesis.

\section{Patterns in multivariate trait space}

We did not detect any multivariate trait space influence of elevation, soil moisture and shade on functional diversity. The overall functional diversity patterns may depend largely on the combination of patterns of many individual traits, which may be masked by opposing niche axes (Spasojevic and Suding 2012). Other studies, such as, Cornwell et al. (2006) also found no difference in the multivariate trait space occupied by species in wet and dry chaparral sites, even though species in drier sites exhibited greater functional trait diversity in water use and transport, and species in wetter sites exhibited greater functional diversity in traits related to stature (Cornwell and Ackerly 2009). Similarly, Spasojevic and Suding (2012) have found non-significant multivariate trait space relationship in alpine tundra on Colorado Rocky Mountain, with significant relationships between for plant height and leaf area functional diversity along a stress-resource environmental gradient.

\section{Conclusions}

We hypothesized that alpine meadow communities are structured by both environmental filtering and competition. Environmental filtering increases trait convergence in stressful environments (e.g., low temperature at high elevations or low soil moisture; Weiher and Keddy 1995, Cornwell et al. 2006), whereas competition (limiting similarity, niche partitioning) prevents coexisting species from being too similar in their traits (Chesson 2000, Cornwell and Ackerly 2009, Spasojevic and Suding 2012). This hypothesis, however, is only partly supported by our results, and we found the opposite, trait diversity increasing with environmental stress for several traits (e.g., functional diversity for SLA increased with elevation). This discrepancy suggest that other processes, such as functional redundancy and facilitation, may also operate driving community assembly in these alpine meadows. In addition, we found a similar response in phylogenetic diversity and functional diversity for plant height in relation to soil moisture, and for SLA to shade. Thus, both plant height and SLA may be important in structuring meadow community evolution. The complex patterns in functional and phylogenetic diversity (e.g., the lack of multivariate response, even though individual traits do change along environmental gradients) highlights that the mechanisms of community assembly cannot be understood by examining a single multivariate trait or phylogenetic diversity index, but rather we need to test functional diversity patterns for individual traits.
Acknowledgments. We thank Dr. S. Zhang, H. Bu, K.Liu, C. Li and J. Cao for their help with field work. The study is supported by the Project of the National Natural Science Foundation of China granted to Guozhen Du (41430749, 41171214) and Xiaoming Shi (41201285), the Special Fund for Agro-scientific Research in the Public Interest granted to Guozhen Du and Wei Qi (201203006), and the Fundamental Research Funds for the Central Universities granted to Wei Qi (lzujbky-2014-94).

\section{References}

Ackerly, D.D. 2003. Community assembly, niche conservatism, and adaptive evolution in changing environment. Int. J. Plant Sci. 164: 165-184.

Ackerly, D.D. 2004. Adaptation, niche conservatism, and convergence: comparative studies of leaf evolution in the California chaparral. Am. Nat. 163(5): 654-671.

Adler, P.B., A. Fajardo, A.R. Kleinhesselink and N.J. Kraft. 2013 Trait-based tests of coexistence mechanisms. Ecol. Lett. 16: 1294-1306.

Bailey-Serres, J. and L.A.C.J. Voesenek. 2008. Flooding stress: acclimations and genetic diversity. Annu. Rev. Plant Biol. 59: 313339.

Baraloto, C., O.J. Hardy, C.E. Paine, K.G. Dexter, C. Cruaud, L.T. Dunning, M.-A. Gonzalez, J.-F. Molino, D. Sabatier, V. Savolainen and J. Chave. 2012. Using functional traits and phylogenetic trees to examine the assembly of tropical tree communities. J. Ecol. 100: 690-701.

Baumeister, D. and R.M. Callaway. 2006. Facilitative effects of Pinus flexilis during succession: a hierarchy of mechanisms benefits other plant species. Ecology 87: 1816-1830.

Bernard-Verdier, M., M.L. Navas, M. Vellend, C. Violle, A. Fayolle and E. Garnier. 2012. Community assembly along a soil depth gradient: contrasting patterns of plant trait convergence and divergence in a Mediterranean rangeland. J. Ecol. 100(6): 14221433.

Bryant, J.A., C. Lamanna, H. Morlon, A.J. Kerkhoff, B.J. Enquist and J.L. Green. 2008. Microbes on mountainsides: contrasting elevational patterns of bacterial and plant diversity. Proc. Natl. Acad. Sci. USA 105 (S1): 11505-11511.

Carboni, M., A.T. Acosta and C. Ricotta. 2013. Are differences in functional diversity among plant communities on Mediterranean coastal dunes driven by their phylogenetic history? J. Veg. Sci. 24(5): 932-941.

Chesson, P. 2000. Mechanisms of maintenance of species diversity. Annu. Rev. Ecol. Syst. 31: 343-366.

Cornwell, W.K. and D.D. Ackerly. 2009. Community assembly and shifts in the distribution of trait values across an environmental gradient in coastal California. Ecol. Monogr. 79: 109-126.

Cornwell, W.K., D.W. Schwilk and D.D. Ackerly. 2006. A trait-based test for habitat filtering: convex hull volume. Ecology 87: 14651471.

Crisp, M.D., M.T.K. Arroyo, L.G. Cook, M.A. Gandolfo, G.J. Jordan, M.S. McGlone, P.H. Weston, M. Westoby, P. Wilf and H.P. Linder. 2009. Phylogenetic biome conservation on a global scale. Nature 458: 754-758.

CVEC, CAS (The China Vegetation Editorial Committee, Chinese Academy of Sciences). 1980. Vegetation of China. Science, Beijing. 
Du, G. and W. Qi. 2010. Trade-offs between flowering time, plant height, and seed size within and across 11 communities of a QingHai-Tibetan flora. Plant Ecol. 209: 321-333.

Dornelas, M., S.R. Connolly and T.P. Hughes. 2006. Coral reef diversity refutes the neutral theory of biodiversity. Nature 440: 80-82.

Duarte, L.S. 2011. Phylogenetic habitat filtering influences forest nucleation in grasslands. Oikos 120: 208-215.

Freckleton, R.P., P.H. Harvey and M. Pagel. 2002. Phylogenetic analysis and comparative data: a test and review of evidence. Am. Nat. 160: 712-726.

Gibbs, J. and H. Greenway. 2003. Review: Mechanisms of anoxia tolerance in plants. I. Growth, survival and anaerobic catabolism. Funct. Plant Biol. 30: 353-353.

Grime, J.P. 2006. Trait convergence and trait divergence in herbaceous plant communities: mechanisms and consequences. J. Veg. Sci. 17: 255-260.

Gross, N., T.M. Robson, S. Lavorel, C. Albert, L. Bagousse-Pinguet and R. Guillemin. 2008. Plant response traits mediate the effects of subalpine grasslands on soil moisture. New Phytol. 180: 652662.

Hakes, A.S. and J.T. Cronin. 2011. Environmental heterogeneity and spatiotemporal variability in plant defense traits. Oikos 120(3): 452-462.

Harmon, L., J. Weir, C. Brock, R. Glor, W. Challenger, G. Hunt, R. FitzJohn, M. Pennell, G. Slater, J. Brown, J. Uyeda and J. Eastman. 2014. GEIGER: analysis of evolutionary diversification. R package version 2.0.3. Available at http://cran.r-project. org/package $=$ geiger.

Hubbell, S.P. 2001. The Unified Neutral Theory of Biodiversity and Biogeography. Princeton University Press, Princeton, New Jersey, USA.

Karst, J., B. Gilbert and M.J. Lechowicz. 2005. Fern community assembly: the roles of chance and the environment at local and intermediate scales. Ecology 86: 2473-2486.

Katabuchi, M., H. Kurokawa, S.J. Davies, S. Tan and T. Nakashizuka. 2012. Soil resource availability shapes community trait structure in a species-rich dipterocarp forest. J. Ecol. 100(3): 643-651.

Kembel, S.W. and S.P. Hubbell. 2006. The phylogenetic structure of a neotropical forest tree community. Ecology 87: S86-S99.

Klein, J.A., J. Harte and X. Zhao. 2007. Experimental warming, not grazing, decreases rangeland quality on the Tibetan Plateau. Ecol. Appl. 17: 541-557.

Kraft, N.J.B., W.K. Cornwell, C.O. Webb and D.D. Ackerly. 2007. Trait evolution, community assembly, and the phylogenetic structure of ecological communities. Am. Nat. 170: 271-283.

Kluge, J. and M. Kessler. 2011. Phylogenetic diversity, trait diversity and niches: species assembly of ferns along a tropical elevational gradient. J. Biogeog. 38: 394-405.

Lavorel, S., K. Grigulis, S. McIntyre, N.S.G. Williams, D. Garden, J. Dorrough, S. Berman, F. Quetier, A. Thebault and A. Bonis. 2008. Assessing functional diversity in the field-methodology matters! Funct. Ecol. 22: 134-147.

Lebrija-Trejos, E., E.A. Perez-Garcia, J.A. Meave, F. Bongers and L. Poorter. 2010. Functional traits and environmental filtering drive community assembly in a species-rich tropical system. Ecology 91: 386-398.

Leibold, M.A., E.P. Economo and P. Peres-Neto. 2010. Metacommunity phylogenetics: separating the roles of environmental filters and historical biogeography. Ecol. Lett. 13: 12901299

Lewis, R.J., R.H. Marrs and R.J. Pakeman. 2014. Inferring temporal shifts in landuse intensity from functional response traits and functional diversity patterns: a study of Scotland's machair grassland. Oikos 123: 334-344.

Li, X. X. Zhu, Y. Niu and H. Sun. 2014. Phylogenetic clustering and overdispersion for alpine plants along elevational gradient in the Hengduan Mountains Region, southwest China. J. Syst. Evol. 52 (3): $280-288$.

Lloret, F., F. Médail, G. Brundu and P. Hulme. 2004. Local and regional abundance of exotic plant species on Mediterranean islands: are species traits important? Global Ecol. Biogeogr. 13: 37-45.

MacArthur, R.H. and R. Levins. 1967. The limiting similarity, convergence, and divergence of coexisting species. Am. Nat. 101: 377-385.

McGill, B.J., B.J. Enquist, E. Weiher and M. Westoby. 2006. Rebuilding community ecology from functional traits. Trends Ecol. Evol. 21: 178-185.

Myers, J.A. and K. Kitajima. 2007. Carbohydrate storage enhances seedling shade and stress tolerance in a neotropical forest. $J$. Ecol. 95: 383-395.

Norden, N., S.G. Letcher, V. Boukili, N.G. Swenson and R. Chazdon. 2012. Demographic drivers of successional changes in phylogenetic structure across life-history changes in plant communities. Ecology 93: S70-S82.

Pagel, M. 1999. Inferring the historical patterns of biological evolution. Nature 401: 877-884.

Pillar, V.D., L.D.S. Duarte, E.E. Sosinski and F. Joner. 2009. Discriminating trait-convergence and trait-divergence assembly patterns in ecological community gradients. J. Veg. Sci. 20: 334348.

Qi, W., S. Guo, X. Chen, J.H.C. Cornelissen, H. Bu, G. Du, X. Cui, W. Li and K. Liu. 2014a. Disentangling ecological, allometric and evolutionary determinants of the relationship between seed mass and elevation: insights from multiple analyses of 1355 angiosperm species on the eastern Tibetan Plateau. Oikos 123: 23-32.

Qi, W., H. Bu, K. Liu, W. Li, J.M. Knops, J. Wang, W. Li and G. Du. 2014b. Biological traits are correlated with elevational distribution range of eastern Tibetan herbaceous species. Plant Ecol. 215: 1187-1198.

R Development Core Team. 2010. R: A Language and Environment for Statistical Computing. $R$ Foundation for Statistical Computing, Vienna, Austria, ISBN 3-900051-07-0. Available at http://www.R-project.org.

Ricklefs, R.E. 2004. A comprehensive framework for global patterns in biodiversity. Ecol. Lett. 7: 1-15.

Ricotta, C. and M. Moretti. 2011. CWM and Rao's quadratic diversity: a unified framework for functional ecology. Oecologia 167: 181-188.

Schluter, D. 2000. Ecological character displacement in adaptive radiation. Am. Nat. 156: S4-S16.

Silvertown, J., M. Dodd, D. Gowing, C. Lawson and K. McConway. 2006. Phylogeny and the hierarchical organization of plant diversity. Ecology 87: S39-S49.

Spasojevic, M.J. and K.N. Suding. 2012. Inferring community assembly mechanisms from functional diversity patterns: the importance of multiple assembly processes. J. Ecol. 100: 652-661.

Swenson, N.G., P. Anglada-Cordero and J.A. Barone. 2011. Deterministic tropical tree community turnover: evidence from patterns of functional beta diversity along an elevational gradient. Proc. R. Soc. B 278: 877-884

Tirado, R. and F.I. Pugnaire. 2005. Community structure and positive interactions in constraining environments. Oikos 111: 437-444. 
Valiente-Banuet, A. and M. Verdú. 2007. Facilitation can increase the phylogenetic diversity of plant communities. Ecol. Lett. 10: 1029-1036.

Valladares, F. and Ü. Niinemets. 2008. Shade tolerance, a key plant feature of complex nature and consequences. Annu. Rev. Ecol. Evol. S. 39:237-257.

Vamosi, S.M., S.B. Heard, J.C. Vamosi and C.O. Webb. 2009 Emerging patterns in the comparative analysis of phylogenetic community structure. Mol. Ecol. 18: 572-592.

Verdú, M., P.J. Rey, J.M. Alcántara, G. Siles and A. Valiente-Banuet. 2009. Phylogenetic signatures of facilitation and competition in successional communites. J. Ecol. 97: 1171-1180.

Wang, Z., Z. Tang and J. Fang. 2007. Altitudinal patterns of seed plant richness in the Gaoligong Mountains, south-east Tibet, China. Divers. Distrib. 13: 845-854.

Webb, C.O., D.D. Ackerly, M.A. McPeek and M.J. Donoghue. 2002. Phylogenies and community ecology. Annu. Rev. Ecol. Syst. 33: 475-505.

Webb, C.O., D.D. Ackerly and S.W. Kembel. 2008. Phylocom: software for the analysis of phylogenetic community structure and trait evolution. Bioinformatics 18: 2098-2100.

Weiher, E. and P.A. Keddy. 1995. Assembly rules, null models, and trait dispersion: new questions from old patterns. Oikos 74: 159165.

Weiher, E., G.D.P. Clarke and P.A. Keddy. 1998. Community assembly rules, morphological dispersion, and the coexistence of plant species. Oikos 81: 309-322

Westoby, M. 1998. A leaf-height-seed (LHS) plant ecology strategy scheme. Plant Soil 199: 213-227.

Westoby, M., D.S. Falster, A.T. Moles, P.A. Vesk and I.J. Wright 2002. Plant ecological strategies: some leading dimensions of variation between species. Annu. Rev. Ecol. Syst. 33: 125-159.

Westoby, M. and I.J. Wright. 2006. Land-plant ecology on the basis of functional traits. Trends Ecol. Evol. 21: 261-268.

Wiens, J. J. and C.H. Graham. 2005. Niche conservatism: integrating evolution, ecology, and conservation biology. Annu. Rev. Ecol. Evol. S. 36: 519-539.
Wikström, N., V. Savolainen and M.W. Chase. 2001. Evolution of the angiosperms: calibrating the family tree. Proc. R. Soc. B 268: $2211-2220$

Willis, C.G., M. Halina, C. Lehman, P.B. Reich, A. Keen, S. McCarthy and J. Cavender-Bares. 2010. Phylogenetic community structure in Minnesota oak savanna is influenced by spatial extent and environmental variation. Ecography 33(3): 565-577.

Wu, C.Y. and P.H. Raven (eds). 1994-2013. Flora of China. Science Press and Missouri Botanical Garden Press, Beijing and St. Louis

Yang, Z., R.P. Jeff, C. Zhang and G. Du. 2012. The effect of environmental and phylogenetic drivers on community assembly in an alpine meadow community. Ecology 93: 2321-2328.

Received April 15,2014

Revised June 19, August 13, 2014 Accepted September 3, 2014

\section{Supplemental material}

\section{Tables S1-S2.}

Figure S1. PCA ordination diagram of seven climate attributes. Abbreviations of climate attributes are as specified in Table S1.

Figure S2. Mean $( \pm 95 \mathrm{CI})$ standardized effect size (SES) of Rao quadratic diversity $(Q)$ for each L-H-S trait and multivariate traits and of phylogenetic diversity in the Tibetan meadow communities. Symbols in black indicate values significantly different from zero.

Appendix 1. A comparison of location of the 57 study sites.

Appendix 2. The methods of L-H-S trait measurement.

Appendix 3. A list of L-H-S traits of 271 species recorded from studied quadrats. SLA, specific leaf area; $\mathrm{PH}$, plant height; SM, seed mass; *, missing values.

The file may be downloaded from www.akademiai.com. 